\title{
LA INVESTIGACIÓN EN CIENCIAS LABORALES COMO UNA NUEVA DIMENSIÓN DEL ALUMNADO UNIVERSITARIO. UNA EXPERIENCIA INNOVADORA
}

\author{
Ricard Calvo Palomares \\ JuAn A. Rodríguez del Pino \\ Raúl Lorente Campos \\ M. Amparo Benedito Monleón \\ Santiago Cantarero Sanz \\ Francisco J. Cano Montero \\ IgNASi LERMa MonTERo
}

INVESLAB (Grupo de Innovación e Investigación en Ciencias Laborales)

Universitat de València

DOI: 10.1387/lan-harremanak.15435

\section{ABSTRACT}

Profesorado del Grado de Relaciones Laborales y Recursos Humanos de la Universitat de València, se planteó en el curso 2013-2014, y dado que la titulación habia pasado de Diplomatura a Grado, la necesidad de fomentar entre el alumnado la orientación investigadora, opción hasta dicho momento dificil de desarrollar. Por eso, a través del desarrollo del proyecto de innovación en dicho curso "Medios para la difusión de resultados de investigaciones en Ciencias Laborales», se creó una web (http://inveslab.blogs.uv.es/) en la que se recogieron los resultados obtenidos del mismo, un repositorio con los medios existentes para la difusión de los resultados de investigaciones en Ciencias Laborales. Todo ello con el objeto de mejorar la capaci- 
dad de los egresados en RRLL y RRHH, de promover la difusión de los resultados y hallazgos obtenidos, procedentes de investigaciones vinculadas con las Ciencias Laborales y, más concretamente, desarrolladas en sus trabajos de fin de Grado (TFG) y/o de fin de Master (TFM). El curso siguiente, 2014-2015, en el que nos encontramos, se ha buscado dar un pequeño paso más con la "Constitución de una red de innovación docente específica en Ciencias Laborales» entre las Universidades de Valencia, Huelva y Murcia (con la intención de ampliarse paulatinamente a otras), con el propósito final de establecer para el próximo curso, 2015-2016, un premio a nivel nacional que estimule las investigaciones realizadas en Ciencias Laborales mediante los trabajos de fin de Grado (TFG) y/o de fin de Master (TFM). Observando la complementariedad e interactuación entre las Universidades españolas y entendiendo a la idea fundamental de mejorar la capacidad divulgadora de los resultados $y$ hallazgos cientificos obtenidos por ellos en las investigaciones realizadas en la materia. En el presente texto realizamos una recopilación del trabajo y de los resultados obtenidos hasta el momento.

Palabras clave: Innovación docente, red de innovación docente, Ciencias Laborales, Relaciones Laborales y Recursos Humanos.

Grade Teacher Labor Relations and Human Resources of the University of Valencia, was raised in the course 2013-2014, and since the degree of Bachelor had been extended from 3 years to 4 years the ability to encourage focused research by students was difficult to develop until this time. Therefore, through the development of the innovation project in the course "Media for the dissemination of research results in Labour Sciences", a website was created (http://inveslab.blogs.uv.es/) in which they collected the results thereof, a repository with existing media to disseminate research results in Labour Sciences. All this in order to improve the ability of graduates in RRLL and RRHH, to promote the dissemination of the results and findings obtained from investigations related to the Labour Sciences and more specifically developed in their final project work (TFG) and / or end of Masters (TFM). The following year, 2014-2015, in which we find ourselves, we have sought to take a small step with the "Constitution of a network of specific teaching innovation in Labour Sciences" between the universities of Valencia, Murcia and Huelva (with the intention to gradually extended to others), with the ultimate aim of establishing for next year, 2015-2016, an award that encourages national research in Labour Sciences by work by Undergraduates (TFG) and / or end Masters (TFM). Noting the complementarity and interaction between the Spanish Universities and understanding the fundamental idea of improving the ability to disclose scientific results and findings obtained by them during field research. In this text we made a compilation of the work and the results obtained so far.

Key Words: Teaching innovation, teaching innovation network, Labour Sciences, Labour Relations and Human Resources. 


\section{Introducción: el por qué de este proyecto}

Los titulados en Ciencias Laborales (Diplomados Graduados Sociales, Diplomados en RRLL, Licenciados en Ciencias del Trabajo y ahora Graduados en RRLL y RRHH) en su proceso de integración y presencia en el mercado de trabajo han tenido un proceso de configuración profesional no exento de dificultades derivadas en gran medida de sus características propias como realidad laboral. Entre ellas destacamos la gran orientación práctica de la misma, lo que ha generado un abandono casi absoluto de su faceta investigadora, no dando a conocer así la relevancia social de su trabajo. Situación que puede verse beneficiada con la reforma universitaria promovida desde el contexto europeo, ya que los planes de estudio han incorporado desde hace unos años la obligatoriedad de cursar determinados créditos ECTS (6 o 12 como es el caso que nos ocupa) vinculados con la investigación. Conocidos en la mayor parte de las titulaciones como Trabajos de Fin de Grado (TFG) o Trabajos de Fin de Máster (TFM) se han convertido en materias con las que los alumnos se han encontrado en sus últimos cursos y que les ha obligado a plantearse la realización de un estudio o investigación sobre alguna materia relacionada con sus estudios de origen.

Dado que muchas de estas investigaciones están siendo de alto interés científico, parece una lástima que los resultados obtenidos en ellas no sean difundidos entre el colectivo profesional y/o la comunidad científica. Como consecuencia, la finalidad del proyecto que se presenta ha sido desarrollar un objeto de aprendizaje que pudiera ser utilizado de forma polivalente en la titulación de 'Grado en Relaciones Laborales y Recursos Humanos', consistente principalmente en la elaboración de unos materiales docentes que pongan de manifiesto los medios existentes en la actualidad para la difusión de los resultados procedentes de investigaciones desarrolladas por egresados en Ciencias Laborales.

\section{Metodología: ¿̨cómo lo hemos desarrollado?}

Para la consecución del objetivo propuesto establecimos un plan de trabajo específico basado en la generación de materiales escritos y audiovisuales suficientes, que permitieran con posterioridad la elaboración de una herramienta infor- 
mática disponible para todo el profesorado con carga docente en Trabajos de Fin de Grado en RRLL y RRHH en el blog http://inveslab.blogs.uv.es/.

Figura 1

Cartel de las Jornadas

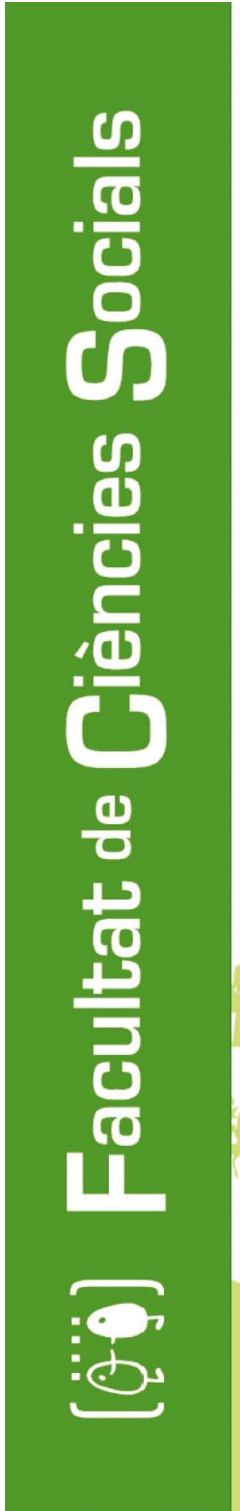

\section{VNIVERSITAT IE VALÈNCIA}

\section{III \\ JORNADAS SOBRE INVESTIGACIÓN SOCIOLABORAL en la Comunitat Valenciana}

"Diseñar, investigar y publicar en Ciencias Laborales: un reto de futuro"

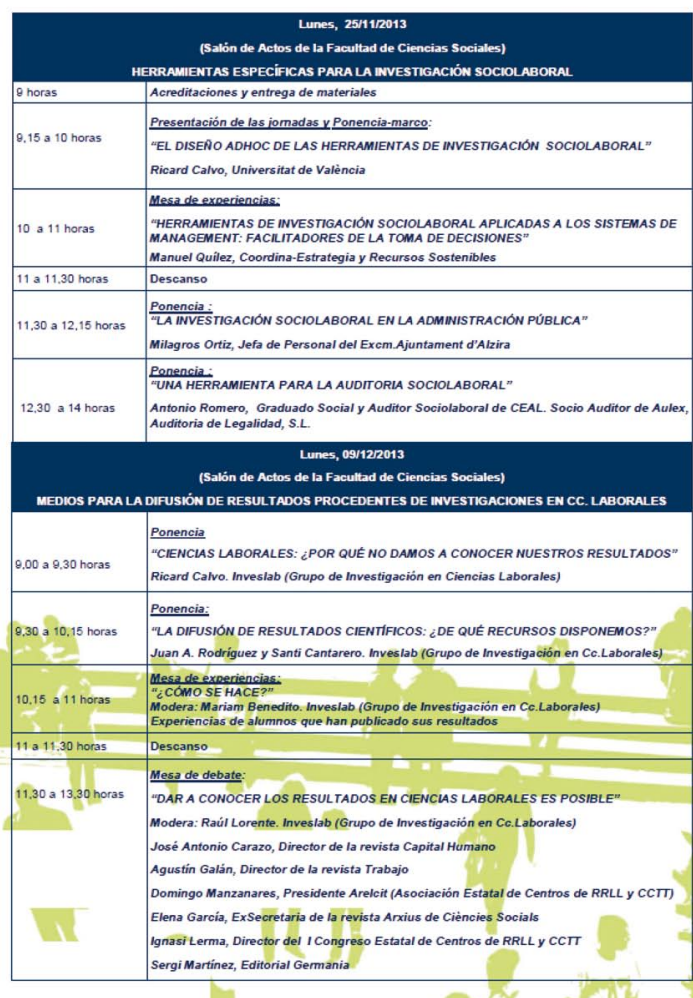

VNIVERSITAT

ID VALĖNCIA $(0)$

Facultat de Ciències Socials

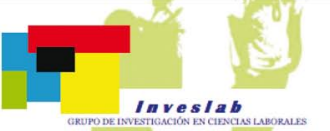

(3) 
Para ello propusimos en primer término realizar una búsqueda de aquellos medios específicos, existentes en la actualidad para la difusión de estudios e investigaciones vinculados con las Ciencias Laborales, de manera periódica (revistas y líneas editoriales) y de manera puntual (congresos y jornadas específicas en la materia). Y en segundo, con la celebración, grabación y posterior análisis de una jornada específica titulada «Medios para la difusión de resultados procedentes de investigaciones en ciencias laborales» (ver figura 1 ).

\section{Los outputs del proyecto: un proceso y un repositorio}

La realización de los TFG, son un requisito formal para la obtención de un título que les faculta para la realización de una actividad laboral. Este requisito puede tener dos concepciones previas: a) la consideración estricta de formal, donde la elaboración del trabajo atiende al cumplimiento de unos mínimos que permita superar su evaluación y obtener el título correspondiente; y, b) la visión de un trabajo con cierta calidad, que reporte tanto en el proceso de ejecución como en su lectura posterior un cierto conocimiento experto relevante, tanto para el que lo realiza como para el que lo evalúa. Esta segunda consideración puede dar lugar a tres tipos de trabajos diferenciados, dependiendo del perfil del alumno, produciendo trabajos de carácter aplicado orientados a la especialización laboral, trabajos más orientados a la actividad investigadora y que puedan dar lugar a estudios superiores centrados en el ámbito de la investigación y, en tercer lugar utilizar la realización de estos trabajos finales para la elaboración de un proyecto emprendedor que permita el diseño y análisis de una idea de negocio.

De estas tres opciones, las dos primeras, además de generar un conocimiento experto para el que lo realiza, puede aportar incluso conocimientos de interés más general, siendo susceptibles de ser evidenciados en alguna publicación técnica o académica, además de su presentación en algún congreso o seminario. La calidad de estos dos tipos de trabajo son consecuencia de una estructura básica para su realización. Se parte de un marco teórico que sustente el planteamiento del alumno, el establecimiento de alguna hipótesis de investigación o la observancia de una teoría en la realidad, para posteriormente establecer alguna metodología empírica o la profundización del conocimiento teórico, que dé lugar a unos resultados que permitan verificar la hipótesis establecida o la revisión de la literatura en un tema concreto.

Si un trabajo consigue tener la calidad suficiente como para aportar conocimiento técnico, el siguiente paso sería plantearse el proceso de publicación o presentación. En esta situación, la dificultad con la que se encuentra el alumno es dónde dirigir su trabajo, dónde enviarlo para que se valore su publicación. 
Y, por otro lado, cómo debe estructurarse el trabajo y su extensión para que sea susceptible de ser valorado por los editores y revisores de la publicación o congreso correspondiente.

En general, los trabajos solicitados por revistas académicas siguen todos una estructura básica (breve introducción, marco conceptual, objeto de estudio, análisis y contrastación de hipótesis, conclusiones finales y bibliografía) y

Figura 2

Repositorio de revistas de Ciencias Laborales

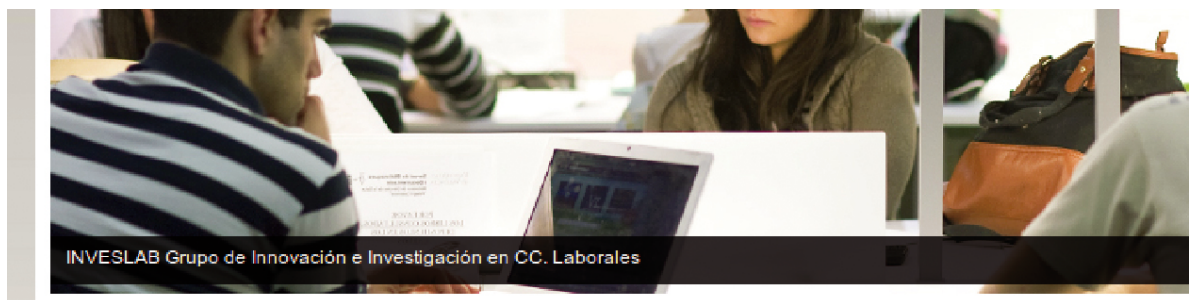

REPOSITORIO REVISTAS Y CONGRESOS-JORNADAS

Abaco. Revista de cultura y ciencias sociales

Acciones e investigaciones sociales

Acta Universitaria

Administración \& desarrollo

Aedipe. Revista de la Asociación Española de Dirección de Persona

Agora. Revista de ciencias sociales

Alba. Revista d'estudis comarcals de la vall d'albaida

Al-Basit

Almenara Revista Fxtremeña de Ciencias Sociales

Alta dirección

Ambitos. Revista de estudias de ciencias sociales y humanidades

Anales de estudios económicos v empresariales

ANDAMIOS. Revista de investigación social

Anduli. Revista andaluza de ciencias sociales

Anuari de L'Agrupacio Borrianenca de Cultura

Anuario da Facultade de Ciencias do Traballo

Aposta. Revista de ciencias sociales

Apuntes. Revista de ciencias sociales

Areas. Revista internacional de ciencias sociales

Arxius de Ciències Socials

Asparkia. Investigacio feminista

Atlanntida. Revista Canaria de Giencias Sociales

Barataria. Revista castellano-manchega de ciencias sociales

Boletín Internacional de Investigación Sindica

Buenaval. Revista de investigación social

Capital humano. Revista para la integración y desarrollo de los recursos humanos

Centro de Estudios del Maestrazgo. Boletin de Divulgacion Cultural

Ciencia $\&$ trabajo

eee/ Ciriec-España. Revista de economia publica, social y cooperativa 
su extensión vendrá marcada por las normas de la publicación (que variará entre 10-20 páginas normalmente). Ello requerirá de un profundo trabajo de síntesis y selección de la información del trabajo, ya que se debe sintetizar la gran extensión del trabajo realizado en unas pocas páginas o en una presentación de poco más de diez minutos. A su vez habrá que plantearse en dónde se enviará, y éste es uno de los grandes problemas, ya que no existe actualmente un repositorio de revistas y congresos que permita la elección del más adecuado para los trabajos realizados en el ámbito de las Ciencias Laborales.

Ante esta tesitura, el proyecto propone como out básico (disponible en http://inveslab.blogs.uv.es/innovacion/repositorio-de-revistas-y-congresosjornadas/), un repositorio de revistas (92 referencias) y congresos y jornadas (13), organizado por áreas temáticas y tipos de publicaciones, desde las más directamente relacionadas con las Relaciones Laborales hasta las menos (ciencias sociales, en general; psicología, etc.).

\section{Reflexiones finales}

\section{a) Un proceso no exento de dificultades}

El desarrollo del proyecto ha puesto de manifiesto que se trata de una necesidad de futuro de la titulación y a la postre de la profesión, aunque también ha puesto de manifiesto algunos de los principales obstáculos que se presentan a este proceso. Entre ellos, uno que tradicionalmente se ha planteado durante muchos ańos, es que los estudios de relaciones laborales no tuvieron una integración en el sistema universitario español, al ser en su origen unos estudios dependientes del Ministerio de Trabajo y que cuando se integraron en el sistema universitario lo hicieron en forma de diplomatura lo que bloqueaba su continuidad hacia estudios superiores ${ }^{1}$. Sin embargo hace ya 15 años que mediante la implantación de la Licenciatura en Ciencias del Trabajo primero y el desarrollo de los estudios de Grado en RRLL y RRHH después, así como los programas de posgrado: másters y doctorados en este ámbito, se ha equiparado la situación de estos estudios con el resto. Por lo tanto a nivel formal esta plena integración de los estudios de RRLL en el ámbito académico es una realidad, otra cosa es que todavía falte profundizar en elementos que eleven el prestigio de la titulación y de su ámbito de conocimiento, así como en la percepción que tienen sobre todo los propios estudiantes de la valía de su título, sus capacidades y competencias. Estos aspectos deberían ser contemplados para, si se nos permite la expresión,

1 Esta situación de los estudios de relaciones laborales en España durante el siglo pasado, a la que no es ajena obviamente la historia de España, contrasta con la posición que ocuparon estos estudios en otros países tanto centroeuropeos como del ámbito anglosajón, donde los estudios de industrial relations tenían una presencia plena en la universidad y en todos los ámbitos académicos. 
incrementar la "autoestima» académica de los propios estudiantes y desarrollar su potencial.

El segundo obstáculo aparece vinculado al anterior y hace referencia a las dificultades que se han planteado, sobre todo en nuestro país, para desarrollar una perspectiva global sobre el área laboral, un enfoque integrado u holístico, que superase el enfoque parcial de las distintas disciplinas ya asentadas y que sumara más en cuanto a riqueza teórica, conceptual y en los instrumentales que permiten acercarse a la realidad empírica, que la simple agregación de las partes. Se han realizado esfuerzos en este sentido con la nueva estructura del Grado que se aleja definitivamente de la orientación claramente desequilibrada de la centralidad de los aspectos jurídicos de anteriores planes de estudio. Potenciando el peso que adquieren las técnicas de investigación social en el currículo actual, que desarrollan todo un conjunto de competencias y habilidades de investigación, fundamental en el tema que tratamos de generar conocimiento. A su vez otro ejemplo que ha tenido por objetivo reforzar esta voluntad de mayor integración de las distintas perspectivas que se ocupan del trabajo hacia un enfoque transdisciplinar, han sido las acciones de la Asociación Estatal de Centros Universitarios de Relaciones Laborales y Ciencias del Trabajo (ARELCIT), principalmente la consolidación de la revista 'Trabajo', así como la organización del I Congreso de Centros Universitarios de RRLL y CCTT en septiembre de 2013 que se diseñó con la clara voluntad de que sirviera de foro de intercambio de conocimiento entre sociólogos, economistas, juristas, psicólogos sociales... Todos estos pasos marcan el camino a seguir en los próximos ańos en este sentido de mayor avance en la transdisciplinariedad del enfoque sobre la realidad laboral.

\section{b) Aportaciones y cuestiones para el debate}

A partir de aquí podemos señalar un conjunto de conclusiones. La primera de ellas hace referencia a las oportunidades que se presentan en el momento actual para avanzar en este proceso de visibilización del área de las ciencias laborales per se o de una forma integrada. El contexto ha cambiado sustancialmente al equipararse plenamente estos estudios en el ámbito universitario al resto de estudios superiores. Si bien los esfuerzos hasta el momento se han centrado en un aspecto fundamental como es la consolidación profesional abriendo nuevos espacios y situando estos estudios en el lugar que naturalmente le corresponde, ocupando entre otros lugares el disputado espacio de los RRHH, también es cierto que un paso adicional fomentando la generación de investigación por parte de los estudiantes y egresados desde el área y su difusión ayudaría en este proceso de consolidación y de poner en valor estos estudios. El currículo actual del Grado lo permite en la medida que integra numerosas materias de técni-

\footnotetext{
2 Anteriormente Trabajo: Revista Andaluza de Relaciones laborales.
} 
cas de investigación en ciencias sociales, y en este sentido se resaltan las ventajas del TFG si se dejase de considerarse por parte del alumnado como una materia más o un obstáculo a superar para obtener el título y pasase a convertirse en una oportunidad que cumpliera la función de "carta de presentación» de los alumnos en la que se mostrase el conjunto de contenidos y competencias adquiridas a lo largo de los estudios. Un trabajo que expresara claramente quien es ese profesional potencial y lo que es capaz de realizar, a modo de ampliación del CV del egresado universitario.

Al mismo tiempo desde ámbitos profesionales se percibe un interés creciente en la sociedad y en las empresas por el ámbito de la organización de las personas y los recursos humanos. En un primer momento este interés se centró en ampliar la gestión de personal a temas como la formación y la motivación de los trabajadores, para ampliarse progresivamente a la gestión por competencias, la gestión integral y temas vinculados al desarrollo, detectándose en los últimos años un renovado interés por los temas de relaciones laborales. A este interés profesional se agrega el claro interés social que tiene la investigación sobre temas laborales en forma de conocimiento que revierta sobre la sociedad para entender e interpretar los procesos sociales, y en general efectuar diagnósticos, comprender las causas de los fenómenos sociales y plantear soluciones. La extensión del desempleo, la precariedad laboral, las formas de trabajo irregular y dimensión de la economía sumergida en nuestro país así como la necesidad de reorientar la actividad productiva y lo que ello implica en términos laborales son claros exponentes de esta necesidad social. Todo ello apunta al interés creciente que debiera suscitar la investigación en el área laboral en todos y cada uno de los niveles, y no solo en los más formalizados y académicos, sino también en fases formativas o niveles profesionales.

La segunda de las conclusiones que podemos extraer una vez confirmadas las potencialidades existentes es la necesidad de escalonar el proceso. Una vez despertado el interés en los estudiantes por la investigación laboral y realizada ésta bajo la guía del tutor y siguiendo un rigor teórico y metodológico, el estudiante debiera plantearse la exposición pública del TFG y su defensa. La experiencia señala que son muy escasos los estudiantes de Grado que optan a la nota máxima por medio de la defensa pública de su trabajo. Un paso posterior sería la participación en congresos por medio de posters o comunicaciones. En este sentido es pertinente desarrollar espacios donde se pueda desarrollar la difusión de estos trabajos bien por medio de áreas específicas para trabajos de estudiantes en los distintos congresos como por medio de congresos específicos para estudiantes ${ }^{3}$. Además obviamente de que se puedan presentar para ser sometidos a evaluación

\footnotetext{
3 Los congresos de la Asociación Estatal de Alumnos y exalumnos de Relaciones laborales pudieran cumplir perfectamente esta función si añadieran a sus facetas más profesionales y reivindicativas una vertiente que incluyera la investigación y difusión de la misma.
} 
directamente en los congresos científicos. Esta participación permitiría a los estudiantes obtener un feedback imprescindible para la mejora de sus trabajos. Destacaríamos aquí la pertinencia de incrementar el número de congresos centrados en el trabajo desde una perspectiva interdisciplinar.

Los premios y concursos de investigación también se muestran como un vehículo idóneo para la difusión de estos materiales ya que la mayor parte de las veces incluyen la publicación de los mismos. Estas convocatorias cada vez son más frecuentes por medio de la difusión de «Olimpiadas», y diversas acciones desarrolladas por Facultades, y otros organismos y fundaciones. El último paso sería plantear la publicación en forma de artículo y en este sentido se constata la posibilidad de publicación de investigaciones laborales de personas más noveles en revistas vinculadas a las facultades donde se imparte la titulación de Grado en RRLL y RRHH, como el caso de Quaderns de Ciències Socials para el caso de la Universitat de València, donde tienen cabida en sus monografías investigaciones de personas que se encuentren en proceso de formación como investigadores. Las revistas de los colegios profesionales, como el colegio de Graduados Sociales, con temáticas más abiertas y aplicadas, donde muchas veces ya se contemplan estudios de caso, constituyen un área potencial donde tuviera cabida la difusión del material de investigación mencionado.

En definitiva, creemos que en el momento actual y una vez superados los obstáculos existentes, se dan las condiciones y la oportunidad para la mejora de la práctica investigadora de los estudiantes en el área de las ciencias laborales y la difusión de esta investigación. 